\title{
ADAPTIVE UNSCENTED TRANSFORM FOR UNCERTAINTY QUANTIFICATION IN EMC LARGE-SCALE SYSTEMS
}

\author{
Moises FERBER*, Christian VOLLAIRE*, Laurent KRAHENBUHL*, \\ and João VASCONCELOS** \\ *Université de Lyon, Laboratoire Ampère (CNRS UMR5005), 69134 Ecully, France \\ E-mail: moisesferber@gmail.com \\ **Universidade Federal de Minas Gerais, Laboratorio de Computação Evolucionaria, \\ 31270-901 Belo Horizonte, Brazil
}

\begin{abstract}
The Unscented Transform (UT) is a stochastic collocation method used for uncertainty quantification in nonlinear systems. This methodology is particularly interesting for EMC models with high-fidelity simulations which are time-consuming, since it demands only a few runs of the electromagnetic solver. However, since the number of simulations required by the UT increases exponentially with the number of dimensions, this methodology becomes unpractical for large-scale systems. Nevertheless, an adaptive Unscented Transform can be an efficient alternative of uncertainty propagation for these large dimensional systems.
\end{abstract}

Keywords: Conducted Interference, Electromagnetic Compatibility, Stochastic, Uncertainty, Unscented Transform

\section{INTRODUCTION}

The recent development of computational tools for electromagnetic compatibility allows accurate simulations of large-scale EMC systems. For instance, a model of a power converter can take into account the intrinsic parasitic effects of components, capacitive and inductive coupling of PCB tracks and nonlinear behavior of semiconductors. Thus, the conducted electromagnetic interference determined using this model is very accurate. However, the computational cost of one simulation is very high.

Many parameters of EMC models are actually known up to a certain precision only. This parametric uncertainty is not taken into account by EMC solvers. One possible approach is to develop a nonintrusive methodology, that uses the results of the solvers for different input scenarios and compute statistics of the output. An example of a methodology that has been applied successfully in EMC is the Unscented Transform (UT) [1] to [4].

Although the UT is much more efficient than Monte Carlo simulations to compute the statistical moments (average, mean, skewness and kurtosis), it is not appropriate for large-scale systems. The required number of simulations increases exponentially with the number of dimensions of the model. For instance, a 10-D model would require at least 2808 simulations to estimate the statistical moments of 1 output variable using $4^{\text {th }}$ order UT approximation [2].

In this context, an adaptive UT for the uncertainty quantification of large-scale EMC models that explores the dominance of some dimensions over others is an interesting alternative to classical UT.

\section{METHODOLOGY AND RESULTS}

\section{Unscented Transform}

The UT consists of estimating the statistical moments of an output variable using the result of wellchosen input values called sigma points $\left(\mathrm{S}_{\mathrm{i}}\right)$. A detailed description of the methodology can be found in [1]. The mean $(\bar{G})$ and the variance ( $\sigma_{G}^{2}$ ), for instance, are given by (1) and (2) respectively.

$$
\begin{aligned}
& \bar{G}=E\{G(\bar{U}+\hat{u})\}=w_{0} G(\bar{U})+\sum_{i=1}^{N} w_{i} G\left(\bar{U}+S_{i}\right) . \\
& \sigma_{G}^{2}=E\left\{(G(\bar{U}+\hat{u})-\bar{G})^{2}\right\}=w_{0}(G(\bar{U})-\bar{G})^{2}+\sum_{i=1}^{N} w_{i}\left(G\left(\bar{U}+S_{i}\right)-\bar{G}\right)^{2} .
\end{aligned}
$$

where $\bar{U}$ is a vector with average input values, $\hat{u}$ is a vector with normalized random input variables and $w_{i}$ for $\mathrm{i}=1$ to $\mathrm{N}$ are weights. 
The sigma points are determined by the solution of the system of nonlinear equations in (3) where $k$ is the order of approximation.

$$
w_{0}=1-\sum_{i} w_{i}, \quad \sum_{i} w_{i} S_{i}^{k}=E\left\{\hat{u}^{k}\right\} .
$$

\section{Adaptive Unscented Transform}

An alternative method to the classical UT is described as follows: rank the input parameters by their influence on the output variable, apply the UT considering only the most important variable and successively one more variable at a time, following the order of importance and stop when convergence is reached. In a largescale system that only a few input parameters must be considered, this adaptive approach is very effective. Figure 1 presents an overview of the adaptive UT.

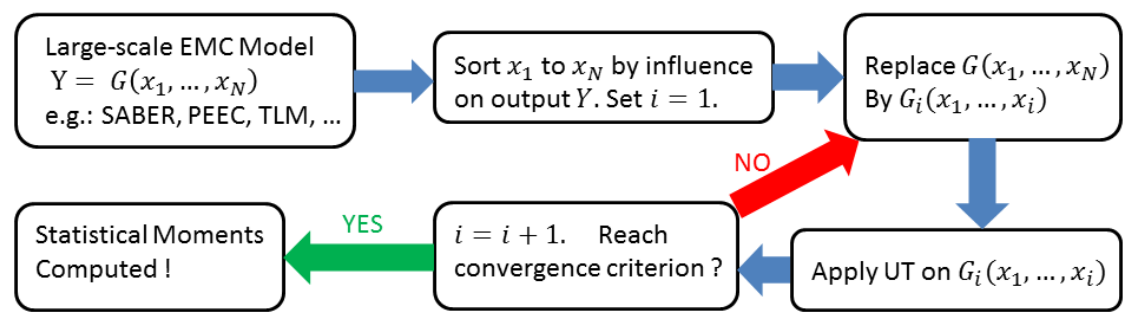

Figure 1. Overview of adaptive unscented transform

\section{Results}

The adaptive UT was applied to a nonlinear model $G$ depicted in (4), and the results presented in Table 1. In the full version of this article, the adaptive UT will be presented in detail and tested with a realistic EMC model. The fast convergence when compared to Monte Carlo will also be shown for different levels of uncertainty and probability density functions.

$$
G(\vec{x})=20 * \log _{10}\left(\sum_{i=1}^{50} a_{i} x_{i}+\sum_{i=1}^{50} a_{i} x_{i}^{2}+\prod_{i=1}^{4} a_{i} x_{i}+\prod_{i=5}^{50} a_{i} x_{i}\right)
$$

where $a_{i}$ for $i=1$ to 4 are coefficients with values 10 times higher than for $\mathrm{i}=5$ to 50 .

Table 1. Comparison between Monte Carlo and Adpative Unscented Transform

\begin{tabular}{|c|c|c|c|}
\hline Methodology & Mean & Variance & \# solver calls \\
\hline Monte Carlo & 78.1528 & 0.7423 & 10000 \\
\hline Adaptive UT & 78.1042 & 0.7963 & 69 \\
\hline
\end{tabular}

\section{CONCLUSIONS}

Collocation methods can be suitable for some large-scale systems. After a sensitivity analysis is used for input variable ranking, an adaptive UT can be applied for uncertainty quantification with fast convergence rate. Higher statistical moments can be computed with higher order UT's. Moreover, the nonintrusive methodology is suitable for coupling the uncertainty algorithm with electromagnetic solvers.

\section{REFERENCES}

[1] S. 1. Julier and 1. K. Ulmann, "Unscented filtering and non-linear estimation", Proc. IEEE, vol. 92(3), pp. 401-422, 2004.

[2] L. de Menezes, A. Ajayi, C. Christopoulos, P. Sewell, G. A. Borges, "Efficient Computation of Stochastic Electromagnetic Problems Using Unscented Transforms", IET Science, Measurement \& Technology, no.2 pp.88-95, 2008.

[3] L. R. A X. de Menezes et ai, "Statistics of the shielding effectiveness of cabinets", in Proc. ESA Workshop on Aerospace EMC, Florence, Session 8, paper 1, 6 pages, 2009.

[4] A Ajayi et al, "Direct computation of statistical variations in EM problems", IEEE Trans. Electromagn. Compat., vol. 50(2), pp. 325332,2008 . 